\title{
Incidencia de ojo seco y úlcera corneal en niños con ventilación mecánica tratados con bloqueo neuromuscular
}

\author{
Incidence of dry eye and corneal ulcer in children with mechanical \\ ventilation treated with neuromuscular blockade
}

\author{
Jesús Javier Martínez-García,*,‡,+ Denisse Rosario Miranda-Hernández, ${ }^{\S}$ Daniel Carlon-Rodrigo『 \\ * Unidad de Terapia Intensiva Pediátrica, Hospital Pediátrico de Sinaloa "Dr. Rigoberto Aguilar Pico". \\ Culiacán, Sinaloa, México; ${ }^{\ddagger}$ Centro de Investigación Aplicada a la Salud Pública. Facultad de Medicina, \\ Universidad Autónoma de Sinaloa; ${ }^{\S}$ Residente de tercer año de Pediatría; ` Oftalmología Pediátrica, \\ Hospital Pediátrico de Sinaloa “Dr. Rigoberto Aguilar Pico”. Culiacán, Sinaloa, México.
}

\section{RESUMEN}

Introducción: El tratamiento con sedación y bloqueo neuromuscular es frecuente en las unidades de cuidados intensivos pediátricos, incrementando el riesgo de ojo seco y úlcera corneal. Objetivo: Determinar la incidencia de ojo seco y úlcera corneal en niños críticamente enfermos con ventilación mecánica que reciben bloqueador neuromuscular. Material y métodos: Estudio de una cohorte prospectiva. Se incluyeron pacientes menores de 18 años con ventilación mecánica que recibieron tratamiento con sedación, analgesia y bloqueador neuromuscular. La evaluación de ojo seco se realizó con prueba de Schirmer I y para úlcera corneal mediante tinción de fluoresceína, lo cual se realizó al momento del inicio de la ventilación, y a las 24,72 y 120 horas posteriores. Todas las mediciones fueron realizadas por el mismo oftalmólogo pediatra. Resultados: Se incluyeron 56 pacientes. A las 24 $\mathrm{h}$ se registraron 11 pacientes con ojo seco, en este tiempo no se documentaron casos con úlcera corneal. A las $72 \mathrm{~h}$, dos pacientes desarrollaron ojo seco y uno úlcera corneal. A las $120 \mathrm{~h}$, dos pacientes más presentaron ojo seco, los cuales desarrollaron úlcera corneal. Así, la incidencia acumulada de ojo seco fue de $26.7 \%(n=15)$ y de úlcera corneal $5.3 \%$ $(n=3)$. Conclusión: En nuestro estudio la incidencia de ojo seco y la úlcera corneal fueron más altas que lo reportado previamente. Por lo anterior, es importante el cuidado y la

\section{ABSTRACT}

Introduction: Treatment with sedation and neuromuscular blockade is common in pediatric intensive care units, thus increasing the risk of dry eye and corneal ulcer. Objective: To determine the incidence of dry eye and corneal ulcer in critically ill children with mechanical ventilation who receive neuromuscular blocker. Material and methods: Prospective cohort study. Patients under 18 years of age with mechanical ventilation who received treatment with sedation, analgesia and neuromuscular blocker were included. The evaluation of dry eye was carried out with the Schirmer I test, and by fluorescein staining for corneal ulcer, which was performed at the time of ventilation initiation, and 24, 72 and 120 hours later. All measurements were made by the same Pediatric Ophthalmologist. Results: Fifty-six patients were included. At $24 \mathrm{~h}, 11$ patients with dry eye were registered; during this time, no cases with corneal ulcer were documented. At $72 \mathrm{~h}$, two patients developed dry eye and one corneal ulcer. At $120 \mathrm{~h}$, two more patients had dry eye, who developed a corneal ulcer. Thus, in total, the cumulative incidence of dry eye was $26.7 \%(n=15)$ and of corneal ulcer, $5.3 \%(n=3)$. Conclusion: In our study, the incidence of dry eye and corneal ulcer were higher than previously reported. Therefore, it is always important that the eyes of patients with mechanical ventilation and

+Correspondencia: JJMG, jjmtz64@hotmail.com

Conflicto de intereses: Los autores declaran que no tienen.

Citar como: Martínez-García JJ, Miranda-Hernández DR, Carlon-Rodrigo D. Incidencia de ojo seco y úlcera corneal en niños con ventilación mecánica tratados con bloqueo neuromuscular. Rev Mex Pediatr. 2020; 87(6); 207-211. https://dx.doi.org/10.35366/97681 
evaluación ocular en pacientes que requieren ventilación mecánica y reciben bloqueadores neuromusculares.

Palabras clave: Ojo seco, úlcera corneal, ventilación mecánica, bloqueador neuromuscular, incidencia, niños.

\section{INTRODUCCIÓN}

Los niños en estado crítico comúnmente reciben sedación y analgesia para el tratamiento del dolor, procedimientos invasivos y para reducir el estrés en una enfermedad grave. ${ }^{1}$ También es una práctica común el uso de bloqueadores neuromusculares en niños hospitalizados en las unidades de cuidados intensivos, principalmente para facilitar la ventilación mecánica y reducir las demandas de oxígeno. ${ }^{2}$ La parálisis neuromuscular que condicionan estos fármacos es para todos los músculos esqueléticos; en particular, al afectar los músculos oculares, se alteran los mecanismos de defensa, como el cierre de los párpados o el parpadeo. Sin estos mecanismos, las corneas están expuestas al medio externo, con un alto riesgo de sequedad, infección, ulceración, perforación, cicatrización y posiblemente pérdida de la agudeza visual. ${ }^{3}$ La incidencia de ojo seco y de úlceras corneales en pacientes adultos en estado crítico es muy amplia y oscila entre ocho y $60 \%{ }^{4}$ De ambas complicaciones hay poca información en niños, por lo que no se conoce la frecuencia de ojo seco, mientras que para la úlcera corneal en unidades de cuidados intensivos pediátricos se ha reportado hasta en $10 \% .^{5}$

El objetivo de nuestro estudio fue determinar la incidencia de ojo seco y úlcera corneal en niños críticamente enfermos con ventilación mecánica que reciben sedación, analgesia y relajación neuromuscular.

\section{MATERIAL Y MÉTODOS}

Se trató de un estudio de cohorte, prospectivo. Los pacientes fueron incluidos entre enero de 2016 a septiembre de 2017, mediante un muestreo no probabilístico de casos consecutivos. Los criterios de selección fueron: pacientes entre un mes y 18 años de edad que ingresaron a la Unidad de Terapia Intensiva Pediátrica (UTIP), con necesidad de ventilación mecánica, sedación, analgesia y relajación neuromuscular. Cada paciente debería tener prueba de Schirmer $\geq 13 \mathrm{~mm}$ y de fluoresceína negativa al ingreso a la UTIP o durante las primeras cinco horas de ingreso a la UTIP. neuromuscular blockers have appropriate treatment and evaluation.

Keywords: Dry eye, corneal ulcer, mechanical ventilation, neuromuscular blocker, incidence, children.

Se excluyeron a pacientes con lesión corneal o con alteraciones oculares que imposibilitaban el cierre del párpado, como traumatismo craneoencefálico y cirugía craneofacial, así como aquellos con ventilación mecánica $<36$ horas.

Todos los pacientes recibieron medidas de protección ocular, como mantener párpados cerrados y recibir lubricante ocular a base de polietilenglicol, propilenglicol, HP-Guar y vehículo cbp (Systane), cada cuatro horas en cada ojo.

Las evaluaciones de las condiciones de los ojos, tanto al ingreso a la UTIP como las subsecuentes, fueron realizadas por un solo oftalmólogo pediatra. Para determinar la presencia de ojo seco se utilizó la prueba de Schirmer I (Figura 1), la cual mide la secreción de lágrimas. La primera se realizó durante las primeras cinco horas de
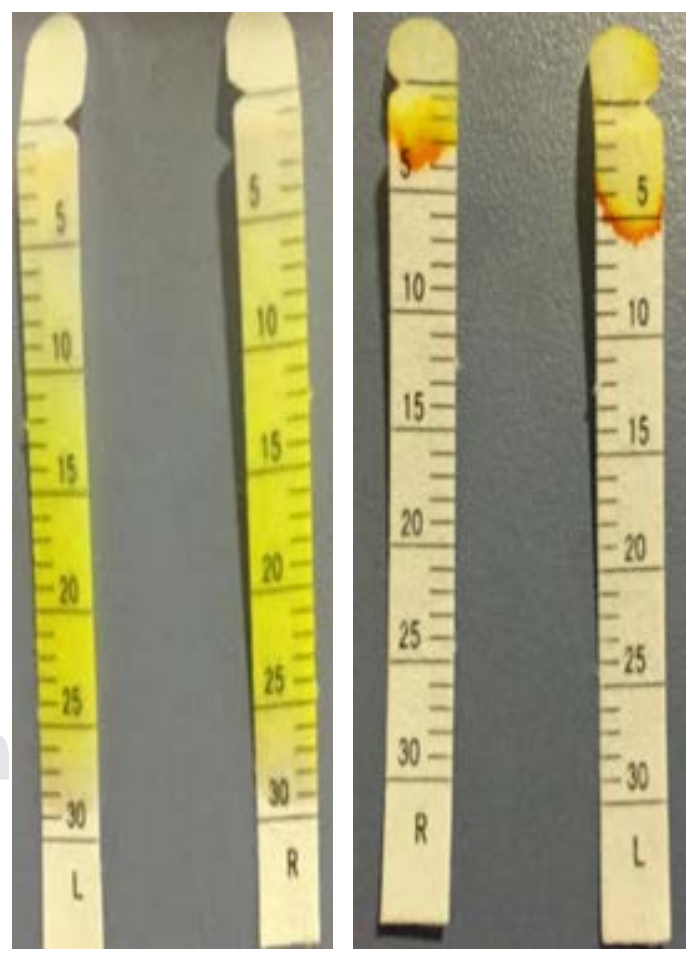

Figura 1: Prueba de Schirmer para evaluar ojo seco: imagen izquierda normal (negativa); imagen derecha positiva (ojo seco). 


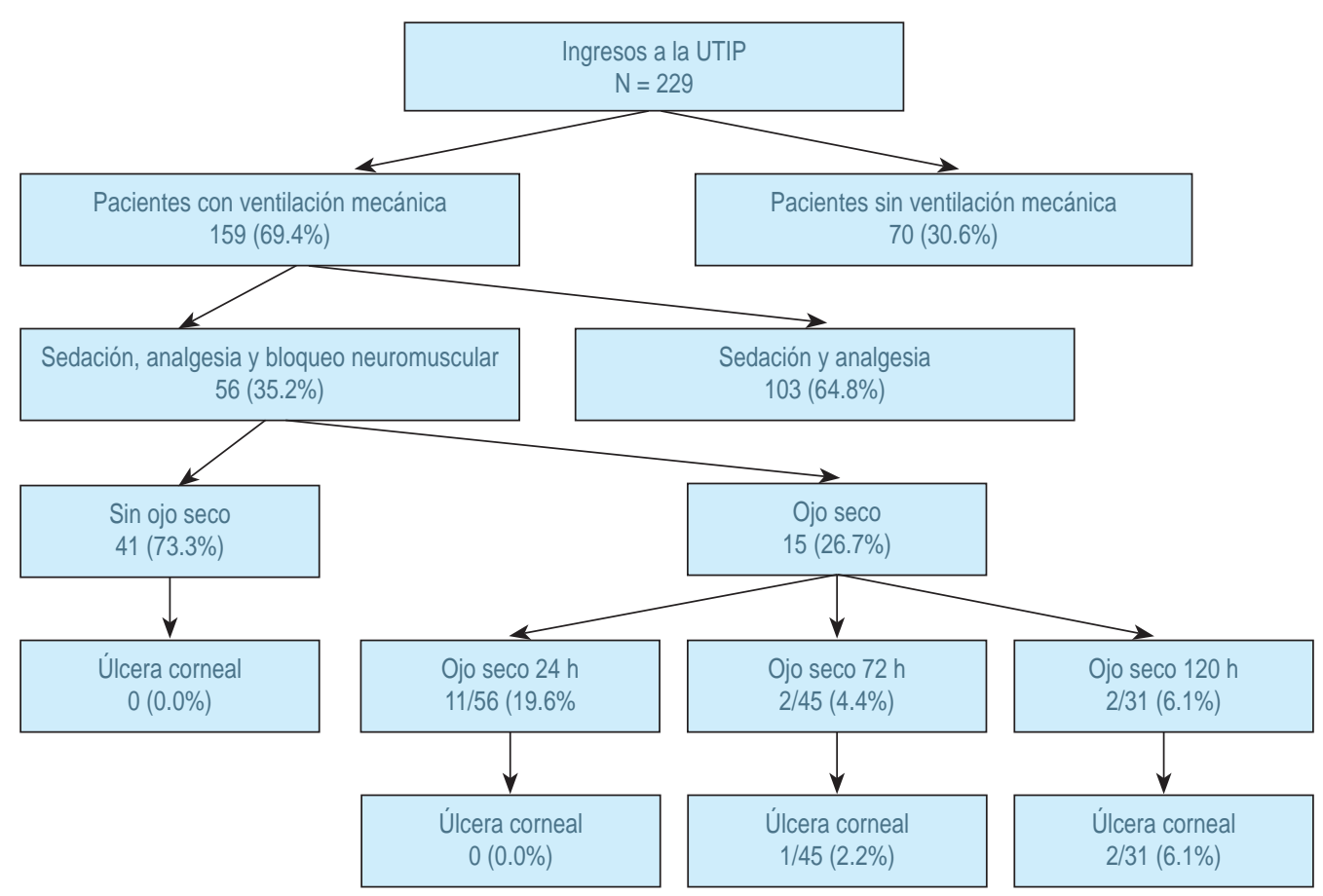

Figura 2: Descripción general de los ingresos a la UTIP y de la cohorte de estudio.

inicio de la ventilación mecánica. Se colocó una tira de Schirmer marca JM ${ }^{\circledR}$ (Schirmer Tear Test Strips, serie 20131204), en el interior del párpado inferior (saco conjuntival), evitando el contacto con la córnea durante cinco minutos. Posteriormente, la tira se retiró y se midió la longitud de la parte húmeda (Figura 1). El diagnóstico de ojo seco fue realizado con un valor $\leq 8 \mathrm{~mm}$ en la tira de Schirmer. ${ }^{6}$ Con respecto a la evaluación de la integridad de la córnea fue mediante tinción de fluoresceína. Para su realización, primero la tira de papel con fluoresceína se impregnó con solución salina y después se aplicó en el fondo del saco conjuntival inferior del ojo sin tocarlo, se cerró el párpado suavemente para cubrir la córnea con fluoresceína y posteriormente el oftalmólogo examinó el ojo con luz azul del filtro (oftalmoscopio Welch Allyn con lente de córnea y luz azul). En su caso, el diagnóstico y tratamiento de úlcera corneal fue determinado por el mismo especialista. Las evaluaciones posteriores se realizaron a las 24,72 y 120 horas.

Las variables de estudio fueron: edad, sexo, peso, diagnóstico principal de ingreso a la UTIP, riesgo de mortalidad pediátrica mediante la escala PRISM III, tipo de sedante, analgésico, relajante neuromuscular y mortalidad.

Análisis estadístico: Se determinó la incidencia de ojo seco y úlcera corneal a las 24, 72 y 120 horas, así como la incidencia acumulada. Debido a que las variables cuantitativas no tuvieron distribución normal, se presentan con mediana, rango intercuartílico (RIC) y valores mínimo y máximo. Mientras que las variables cualitativas con frecuencias simples y porcentajes. Valores de $p<0.05$ se consideraron estadísticamente significativo. Los análisis fueron realizados con el paquete estadístico SPSS v. 22.0, IBM.

Aspectos éticos. El estudio fue aprobado por el comité de ética en investigación del Hospital Pediátrico de Sinaloa "Dr. Rigoberto Aguilar Pico", y bajo consentimiento informado por los padres de los pacientes.

\section{RESULTADOS}

En el periodo de un estudio, 229 pacientes ingresaron a la UTIP, requiriendo $69.4 \%(\mathrm{n}=159)$ de ventilación mecánica. El 35.2\% $(n=56)$ de estos pacientes recibió sedación, analgesia y relajación neuromuscular y fueron los que se incluyeron en el análisis (Figura 2). La mayoría fueron de sexo masculino $(\mathrm{n}=34,60.7 \%)$, la mediana para la edad fue de 8.5 meses (RIC 3.235.5), la mediana de peso fue de $9 \mathrm{~kg}$ (RIC 6.7-13.5). La mediana de puntaje de PRISM III durante las primeras $24 \mathrm{~h}$ fue de 31 (RIC 25-39 puntos). Los diagnósticos principales fueron síndrome de dificultad 
respiratoria aguda ( $\mathrm{n}=24,42.9 \%$ ) y choque séptico (n $=20,35.7 \%)$. Todos los pacientes recibieron sedación con midazolam, analgesia con fentanyl y relajación neuromuscular con atracurio.

La mediana de la prueba de Schirmer basal al ingreso a la UTIP en los 56 pacientes en ojo derecho fue de $15 \mathrm{~mm}$ (RIC 14-20) y, para el ojo izquierdo de $20 \mathrm{~mm}$ (RIC 15-25). Ninguno tuvo lesión corneal.

\section{Incidencia de ojo seco}

Como se muestra en la Figura 2, a las 24 h de ventilación mecánica, la incidencia de ojo seco fue de $19.6 \%$ $(\mathrm{n}=11)$, para estos pacientes, la mediana de la prueba de Schirmer fue de $5 \mathrm{~mm}$ (RIC 3-8). A las $72 \mathrm{~h}, 45$ pacientes seguían con ventilación mecánica, de éstos, en otros dos se detectó ojo seco (4.4\%). En ambos la prueba de Schirmer fue de $5 \mathrm{~mm}$. Mientras que a las $120 \mathrm{~h}, 31$ pacientes permanecían con ventilación mecánica, de los cuales se identificaron dos pacientes más con ojo seco (6.1\%), quienes tuvieron prueba de Schirmer de $5 \mathrm{~mm}$. En total, con los 15 casos detectados, la incidencia acumulada seco en pacientes pediátricos con ventilación mecánica fue de $26.7 \%$ (15/56).

\section{Incidencia de úlcera corneal}

A las 24 h ningún paciente había desarrollado lesión corneal. Para las $72 \mathrm{~h}$, mediante tinción con fluoresceína, se observó úlcera corneal (Figura 3) en una paciente de sexo femenino de dos meses que ingresó por choque séptico por Pseudomonas. A las 120 h otras

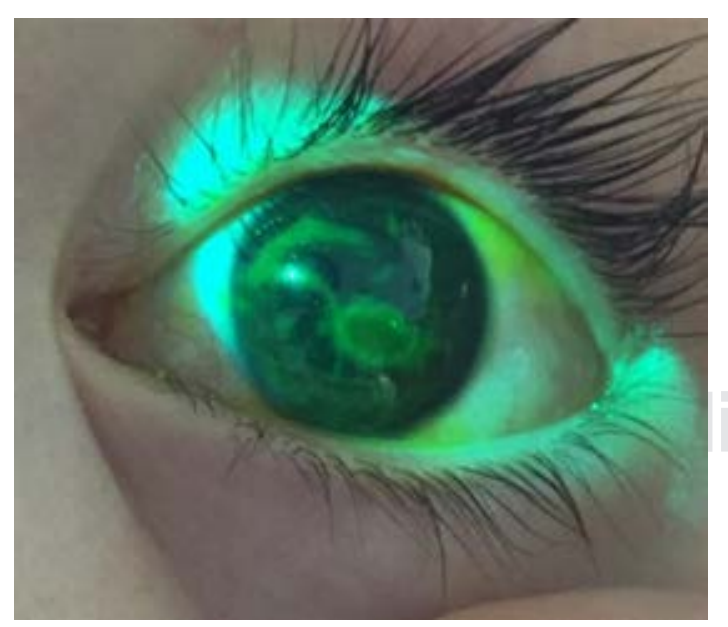

Figura 3: Úlcera corneal mediante evaluación con tinción fluoresceína. dos pacientes del sexo femenino desarrollaron úlcera corneal; la primera tenía cinco años de edad, diagnóstico de apendicitis perforada y sepsis grave. La segunda paciente era de cinco meses de edad con diagnóstico de neumonía y síndrome de dificultad respiratoria aguda. Como se muestra en la Figura 2, en los tres pacientes se había detectado ojo seco previamente. Con estos tres casos, la incidencia acumulada de úlcera corneal fue de $5.3 \%(3 / 56)$.

Cabe hacer mención que el servicio de Oftalmología indicó para estas tres pacientes permanecer con párpados ocluidos y recibir tratamiento con lubricante a base de polietilenglicol, propilenglicol, HP-Guar y vehículo cbp (Systane), carbómero gel (Artelac Nighttime) y gatifloxacina oftálmica. Con estas indicaciones, las pacientes presentaron recuperación completa.

\section{DISCUSIÓN}

La definición actual de ojo seco de acuerdo con The International Dry Eye Workshop la describe como una enfermedad multifactorial de las lágrimas y la superficie ocular que presenta síntomas de malestar, alteraciones visuales e inestabilidad de la película lagrimal, con daño potencial de la superficie ocular. Se acompaña por hiperosmolaridad de la película lagrimal e inflamación de la superficie ocular. ${ }^{6}$ El diagnóstico se puede realizar con la prueba de Schirmer, descrita en 1903, la cual es la prueba más utilizada para evaluar la producción de lágrimas. La prueba de Schirmer I mide la secreción total de lágrimas reflejas y basales. Sin instilar gotas de anestesia, las tiras de Schirmer se insertan en el saco conjuntival inferior temporal, evitando contacto con la córnea. La longitud de humedad se registra en milímetros después de cinco minutos. Aunque los valores normales descritos son variables, se acepta que debe ser $>10 \mathrm{~mm} \cdot{ }^{6-8}$

La incidencia de úlcera corneal en adultos críticamente enfermos es muy amplia, con reportes tan bajos de $8 \%$ y tan altos de $60 \% ., 4$ La incidencia de ojo seco en unidades de cuidados intensivos de adultos reportada por Oh y colaboradores fue de $72.2 \%{ }^{10}$ Negin Masoudi y colegas reportan una incidencia de $32.2 \%$ de ojo seco y $13.8 \%$ de úlcera corneal en una muestra de 87 adultos con ventilación mecánica en Irán; en este estudio se determinó que el ojo seco fue un factor de riesgo para presentar úlcera corneal, ya que el 91.7\% con ojo seco la desarrollaron. ${ }^{11}$ En niños, los reportes sobre la frecuencia de ojo seco y úlcera corneal en unidades de terapia intensiva son limitados. En el 
estudio descrito por Sorce L y su equipo, reportaron una incidencia de $10 \%$ en 207 niños tratados con sedación, analgesia y bloqueador neuromuscular; ${ }^{12}$ sin embargo, no se evaluó la producción de lágrimas, lo cual es importante por la relación causal entre ojo seco y úlcera corneal, ${ }^{10}$ lo cual se comprobó en nuestro estudio (Figura 2).

En el presente estudio hubo limitaciones. Una de ellas es que las evaluaciones de la producción de lágrimas con la prueba Schirmer no se realizaron cada $24 \mathrm{~h}$, y la otra es que el número de pacientes fue limitado.

Por último, es importante tener en cuenta que es necesario el cuidado ocular como parte del tratamiento en el paciente pediátrico en estado crítico, debido a que, con la sedación y relajación neuromuscular, los mecanismos que normalmente participan en la protección del ojo se ven comprometidos. Para evitar el ojo seco y la posibilidad de úlcera cornal se pueden utilizar una variedad de métodos para proteger los ojos en pacientes, tales como el cierre de los párpados, gotas de lágrimas artificiales, gel lubricante, cámara de humedad, así como ungüentos con antibióticos. ${ }^{13,14}$ Sin embargo, parece necesario realizar investigaciones para determinar la efectividad de estos métodos de cuidado.

\section{CONCLUSIÓN}

Dado que la incidencia de ojo seco en niños bajo ventilación mecánica fue de $26.7 \%$, y de úlcera corneal de $5.3 \%$, lo cual es mayor a los reportes previos, pone en relieve la necesidad de brindar manejo preventivo en todos estos pacientes.

\section{AGRADECIMIENTOS}

Al personal de enfermería del Hospital Pediátrico de Sinaloa "Dr. Rigoberto Aguilar Pico".

\section{REFERENCIAS}

1. Lenart SB, Garrity JA. Eye care for patients receiving neuromuscular blocking agents or propofol during mechanical ventilation. Am J Crit Care. 2000; 9: 188-191.

2. McCall K, Hussin HM, Gregory ME, Dutton G, Richardson J. A bundle improves eye care in PICU. Arch Dis Child. 2016; 101: 832-835.

3. Rosenberg JB, Eisen LA. Eye care in the intensive care unit: narrative review and meta-analysis. Crit Care Med. 2008; 36(12): 3151-3155.

4. Kam KYR, Hayes M, Joshi N. Ocular care and complications in the critically ill. Trends Anaesth Crit Care. 2011; 1: 257-262.

5. Suresh P, Mercieca F, Morton A, Tullo AB. Eye care for the critically ill. Intensive Care Med. 2000; 26(2): 162-166.

6. The definition and classification of dry eye disease: report of the Definition and Classification Subcommittee of the International Dry Eye WorkShop (2007). Ocul Surf. 2007; 5(2): 75-92.

7. Li N, Deng XG, He MF. Comparison of the Schirmer I test with and without topical anesthesia for diagnosing dry eye. Int J Ophthalmol. 2012; 5(4): 478-481.

8. Shapiro A, Merin S. Schirmer test and break-up time of tear film in normal subjects. Am J Ophthalmol. 1979; 88(4): 752-757.

9. Zeev MS, Miller DD, Latkany R. Diagnosis of dry eye disease and emerging technologies. Clin Ophthalmol. 2014; 8: 581-590.

10. Oh EG, Lee WH, Yoo JS, Kim SS, Ko IS, Chu SH et al. Factors related to incidence of eye disorders in Korean patients at intensive care units. J Clin Nurs. 2009; 18(1): 29-35.

11. Masoudi Alavi N, Sharifitabar Z, Shaeri M, Adib Hajbaghery M. An audit of eye dryness and corneal abrasion in ICU patients in Iran. Nurs Crit Care. 2014; 19(2): 73-77.

12. Sorce LR, Hamilton SM, Gauvreau K, Mets MB, Hunter DG, Rahmani $\mathrm{B}$ et al. Preventing corneal abrasions in critically ill children receiving neuromuscular blockade: a randomized, controlled trial. Pediatr Crit Care Med. 2009; 10(2): 171-175.

13. Boal C, Corkin D. The importance of protocol-based eye care in the pediatric intensive care unit. Nurs Child Young People. 2019; 31(4): 23-27.

14. Hearne BJ, Hearne EG, Montgomery H, Lightman SL. Eye care in the intensive care unit. J Intensive Care Soc. 2018; 19(4): 345-350. 